\title{
Kein Einfluss auf die kognitive Funktion bei Patienten mit lakunären Schlaganfällen
}

Fragestellung: Können eine antihypertensive Therapie und eine duale Plättchenhemmung bei Patienten mit lakunären Schlaganfällen die kognitiven Funktionen beeinflussen?

Hintergrund: Arterielle Hypertonie und Diabetes mellitus sind wichtige Risikofaktoren für lakunäre Infarkte, aber auch für kognitive Einschränkungen und die vaskuläre Demenz. Theoretisch sollte daher eine konsequente antihypertensive Therapie das Fortschreiten kognitiver Einschränkungen ebenso verhindern wie das Neuauftreten kognitiver Störungen. Ob dies auch für den Einsatz von Thrombozytenfunktionshemmern gilt, wurde bisher nicht gut untersucht. Die SPS3-Studie war eine vom National Institute of Neurological Disorders and Stroke (NINDS) finanzierte Studie in den USA, in der Patienten mit lakunären Infarkten mit zwei unterschiedlichen Therapiezielen antihypertensiv behandelt wurden und bei der eine Monotherapie mit Acetylsalicylsäure und eine Kombinationstherapie für

Pearce LA, McClure LA, Anderson DC et al; SPS3 Investigators. Effects of long-term blood pressure lowering and dual antiplatelet treatment on cognitive function in pa-tients with recent lacunar stroke: a secondary analysis from the SPS3 randomised trial. Lancet Neurol 2014; 13 $1177-85$ Clopidogrel und Acetylsalicylsäure verglichen wurden [1].

Patienten und Methodik: Die SPS3-Studie randomisierte 3.020 Patienten mit einem lakunärem Infarkt innerhalb der letzten sechs Monate in einem $2 \times 2$ faktoriellem Design in zwei Gruppen bezüglich angestrebter Blutdruckwerte von systolisch zwischen 130 und $149 \mathrm{mmHg}$ versus < $130 \mathrm{mmHg}$ und einer plättchenhemmenden Prophylaxe mit einer Monotherapie versus einer dualen Therapie. Als Messinstrument für kognitive Funktionen wurde das kognitive Abilities Screening Instrument (CASI) eingesetzt. Die Patienten in der Studie wurden über einen Zeitraum von fünf Jahren einmal jährlich getestet. Der Unterschied im systolischen Blutdruck zwischen den beiden Therapiegruppen betrug $11 \mathrm{mmHg}$.

Ergebnisse: Die Patienten der Studie waren im Schnitt 63 Jahre alt und $38 \%$ der Studienteilnehmer waren Frauen. Dreiviertel der Patienten wiesen eine arterielle Hypertonie auf und fast alle Patienten hatten einen Barthel-Index von 95 oder höher. Zum Zeitpunkt des Studieneinschlusses bestand bei $45 \%$ der Patienten eine leichte kognitive Einschränkung. 23\% der Patienten hatten deutliche mikroangiopathische Veränderungen in der Kernspintomografie. Über den Zeitraum der Studie veränderten sich die Werte des CASI nicht. Es gab auch keine Interaktion zwischen kognitiven Funktionen und der thrombozytenfunktionshemmenden Therapie oder der beiden Blutdrucktherapiegruppen.

Schlussfolgerungen: Über einen Zeitraum von fünf Jahren spielen die Art der plättchenhemmenden Therapie und das Ausmaß der Blutdrucksenkung bei Patienten mit lakunären Schlaganfällen keine Rolle für das Ausmaß kognitiver Störungen und die Progression dieser Störung.

\section{Die Studiendauer von fünf Jahren war wahrscheinlich zu kurz}

Die SPS3-Studie war negativ bezüglich der Schlaganfallprävention, wenn man die beiden Gruppen mit unterschiedlichen Thrombozytenfunktionshemmern verglich. Daher ist es keine Überraschung, dass sich eine Monotherapie bezüglich kognitiver Funktion nicht von einer dualen Plättchenhemmung unterscheidet. Eher überraschend ist die Beobachtung, dass eine Blutdrucksenkung von durchschnittlich $11 \mathrm{mmHg}$ über einen Zeitraum von fünf Jahren keinen Einfluss auf das Fortschreiten kognitiver Störungen hat. Die wahrscheinlichste Erklärung ist, dass der Zeitraum von fünf Jahren zu kurz ist, um solche Effekte zu sehen. Darüber hinaus waren die meisten Patienten beim Studieneintritt kognitiv intakt oder hatten nur geringe kognitive Einschränkungen. Um den Einfluss einer antihypertensiven Therapie auf die Entwicklung oder das Fortschreiten kognitiver Störungen zu erfassen, müssen wahrscheinlich Studien über einen Zeitraum von mehr als zehn Jahren durchgeführt werden.

\author{
Referenzen: \\ 1. Benavente OR et al. N Engl J Med 2012; 367: 817 - 25
}

Weitere Infos auf

springermedizin.de

Kardioembolischer Schlaganfall

Der Artikel informiert über Symptomatik, Diagnose und

Therapie bei kardioembolischen Schlaganfällen und

ist zu finden, indem Sie den Titel oder die ID-Nummer

(4393114) in die Suche eingeben. 